\title{
Multiple crossing points and possible quantum criticality in the magnetoresistance of thin TiN films
}

\author{
K. Kronfeldner, ${ }^{1}$ T. I. Baturina, ${ }^{1,2}$ and C. Strunk ${ }^{1, *}$ \\ ${ }^{1}$ Institute of Experimental and Applied Physics, University of Regensburg, D-93025 Regensburg, Germany \\ ${ }^{2}$ Institute of Semiconductor Physics, 13 Lavrentjev Avenue, Novosibirsk 630090, Russia
}

(Received 20 July 2020; revised 1 March 2021; accepted 28 April 2021; published 19 May 2021)

\begin{abstract}
We have measured $R(T, B)$ of a TiN thin film very close to the disorder-driven superconductor-insulator transition but still superconducting at zero field and low temperatures. In a magnetic field we find that three distinct crossing points of the magnetoresistance isotherms occur at magnetic fields $B_{c X}$ in three different temperature regions. Each crossing point in $R(T, B)$ corresponds to a plateau in $R\left(T, B_{C X}\right)$. We systematically study the evolution of these crossing points near the disorder-induced superconductor/insulator transition, identify the most promising candidate for a quantum phase transition, and provide estimates for the two critical exponents $z$ and $\nu$.
\end{abstract}

DOI: 10.1103/PhysRevB.103.184512

\section{INTRODUCTION}

Quantum phase transitions (QPTs) occur between competing ground states of many-body systems and result from quantum fluctuations near quantum critical points [1]. The superconductor/insulator transition (SIT) in thin metal films is viewed as one of the prime examples of a QPT [2-4]. It can be driven by one or more control parameters, such as the level of disorder in the system [5] or the electron density $n$ [6]. More generally, the relevant control parameter for the disorder-driven SIT is $\Delta=k_{F}(n) \ell$, where $k_{F}(n)$ is the Fermi wave vector and $\ell$ the mean free path. For $\Delta \gtrsim \Delta_{c}$, the transition can also be driven by a perpendicular magnetic field $B$ [7]. In the limit of zero temperature, the discrimination between superconductor and insulator appears to be trivial, as in the first case the resistance should be zero and in the other infinity. At the experimentally accessible finite temperatures, it has become common practice to regard the sign of the temperature derivative $\partial R(T, B) / \partial T$ of the resistance $R$ for the discrimination of the two ground states.

According to the theory of phase transitions, in a given universality class and near the critical point all properties depend in a universal way on a characteristic length $\xi \propto\left|\Delta-\Delta_{c}\right|^{v}$ with the critical exponent $v$, and a characteristic frequency $\omega \propto \xi^{-z} ; z$ being the dynamical critical exponent. If $R(T, B)$ is controlled by the two critical exponents, the scaling relation $R(T, B) \propto\left|B-B_{c}\right| / T^{1 / z v}$ is expected to hold in the vicinity of the crossing point [3]. In a magnetic field distinct crossing points of the magnetoresistance isotherms $R(T=$ const, $B)$ at a critical magnetic field $B_{c}$ were observed [7-12]. Reversing Fishers' argument, the observed scaling property of $R(T, B)$ is often taken as a signature for critical behavior near a QPT and the crossing points were interpreted as evidence for a magnetic field-induced SIT.

\footnotetext{
*christoph.strunk@physik.uni-regensburg.de
}

The existence of a crossing point in $R(T=$ const, $B)$ implies that $R\left(T, B_{c}\right)=$ const, i.e., a plateau in $R\left(T, B_{c}\right)$. Experimentally, the latter condition is obeyed over finite $T$ intervals only. In addition, more than one crossing point was observed and interpreted as multistage criticality [13-15]. On the other hand, plateaus in $R(T, B=$ const $)$ can be generated by two or more contributions to resistivity with similar $T$ dependence, but opposite sign, leading to a cancellation of the $T$ dependence within a certain range. For example, the positive contribution of superconducting fluctuations to $R(T, B=$ const) can be approximately compensated over a certain $T$ interval by the negative contribution from localization or density of state effects [16-18]. Hence the basic question arises, how to ensure that an observed crossing point indeed signals true quantum critical behavior.

Here, we demonstrate that the scaling property is insufficient, as at least two control parameters are needed to establish a crossing point as a candidate quantum critical point for the magnetic field-induced superconductor/insulator transition. In Fig. 1 we show that the separatrix $B^{*}(T)$ (yellow strips), which separates regimes with positive and negative $\partial R(T, B) / \partial T$, can be strongly nonmonotonic with three extrema of $B^{*}(T)$. Each extremum defines a $T$ interval displaying a distinct crossing of the $R(B)$ isotherms, i.e., a crossing point. We investigate the evolution of critical parameters with the level of disorder and argue that only one of the three crossing points remains as a candidate for the magnetic field-induced SIT.

\section{EXPERIMENTAL DETAILS}

The approximately square shaped samples are patterned on two chips $(\mathrm{A}, \mathrm{B})$ from the same $d \approx 3.6-\mathrm{nm}$ thin TiN film (wafer D03 in [19]). The film on chip A had size $1 \mathrm{~mm} \times$ $2 \mathrm{~mm}$, while chip B had square shaped devices of side length 90 and $240 \mu \mathrm{m}$. All devices were contacted on chip with 100-nm-thick Au films on top of the TiN layer. In this work 


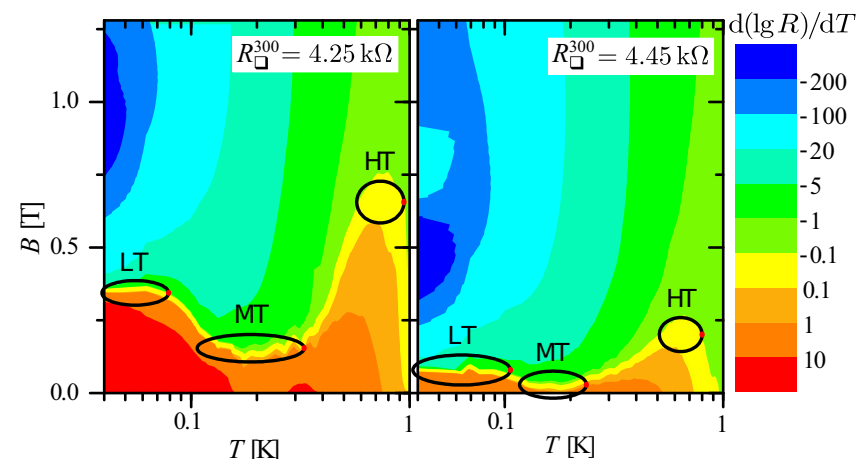

FIG. 1. Logarithmic derivative of $R(T, B)$ vs temperature $T$ and magnetic field $B$ (chip A). Blue/green areas indicate an insulating $(\partial R / \partial T<0)$ trend; red/orange areas indicate a superconducting $(\partial R / \partial T>0)$ trend. The nonmonotonic yellow strip displays the separatrix $B^{*}(T)$ between superconducting and insulating regimes. The three extrema in $B^{*}(T)$ are marked with black ellipses labeled LT, MT, and HT. The two panels display states with different $R_{\square}^{300}$, i.e., different levels of disorder (see text).

we systematically tuned $R_{\square}^{300}$ towards the disorder-driven SIT by stepwise heating in air $\left(\approx 250^{\circ} \mathrm{C}\right.$ for several minutes). In the quasi-two-dimensional (quasi-2D) limit, sheet resistance at room temperature $R_{\square}^{300}$ provides a reliable measure for the level of disorder for different film thicknesses and different oxidation states [20]. The current-voltage $(I-V)$ characteristics are highly nonlinear forcing us to always identify the linear regime of $I(V)$ to extract correct resistance values. We use a heavily filtered voltage biased two-point circuit with an $I-V$ converter to measure $I(V)$ at different temperature and perpendicular magnetic field. The backaction noise from the $I-V$ converter was suppressed by an $R C$ filter in the bias line. The lead $(\simeq 6 \Omega)$ and bias $(\simeq 7-20 \mathrm{k} \Omega)$ resistances were subtracted.

\section{THREE CROSSING POINTS}

The three extrema in $B^{*}(T)$ correspond to plateaus labeled LT, MT, and HT in the $R(T)$ dependence shown in Fig. 2(a), from which the data in Fig. 1 is derived. The corresponding magnetoresistance isotherms at first sight do not reveal well defined crossings. However, when we plot $R(B)$ in Figs. 2(b)2(d) separately for the three plateau regions in Fig. 2(a), three distinct crossing points become clearly visible. In the Supplemental Material [20] we propose empirical expressions for the functional form of the $R(T, B)$ curves.

Each of the crossing points allows for a scaling analysis $[3,21]$. The result is shown in the insets of Figs. 2(b)-2(d). In the LT and MT regimes, we obtained a best data collapse of the $R(T, B)$ data with the scaling exponent $z v=1.2 \pm 0.2$, consistent with the findings in, e.g., indium oxide $[8,22]$ and $a$-MoGe [7]. A smaller value of $z v=0.33 \pm 0.03$ is found in the HT regime. The fact that we observe several such points may even suggest multistage quantum criticality $[13,14]$. This immediately raises the question regarding the nature of the different stages [16].

In order to identify crossing points as candidates for quantum criticality, we follow the evolution of the critical parameters $R_{c}, B_{c}$, and $z v$ as a function of the second nonthermal control parameter, i.e., the level of disorder, quantified by the normal-state resistance $R_{\square}^{300}$. We measured the $R(B)$ isotherms of TiN films with different oxidation states and perform the scaling analysis for each of them. The evolution of the critical parameters vs $R_{\square}^{300}$ is depicted in Fig. 3. Important features in Fig. 3 are the disorder-independent values for $R_{c M} \approx h / 4 e^{2}$ [Fig. 3(a)] and $z v \approx 1.2$ [Fig. 3(b)] in the MT regime. The critical magnetic fields $B_{c}$ for all three crossing points nicely extrapolate to zero at $R_{\square}^{300} \approx 4.5 \mathrm{k} \Omega$, which we associate with the critical level of disorder required for the disorder-induced SIT at $B=0$. According to Fisher [3], the transition field $B_{c}$ vanishes as $\xi^{-2}$ at the disorder-induced SIT at $B=0$. The
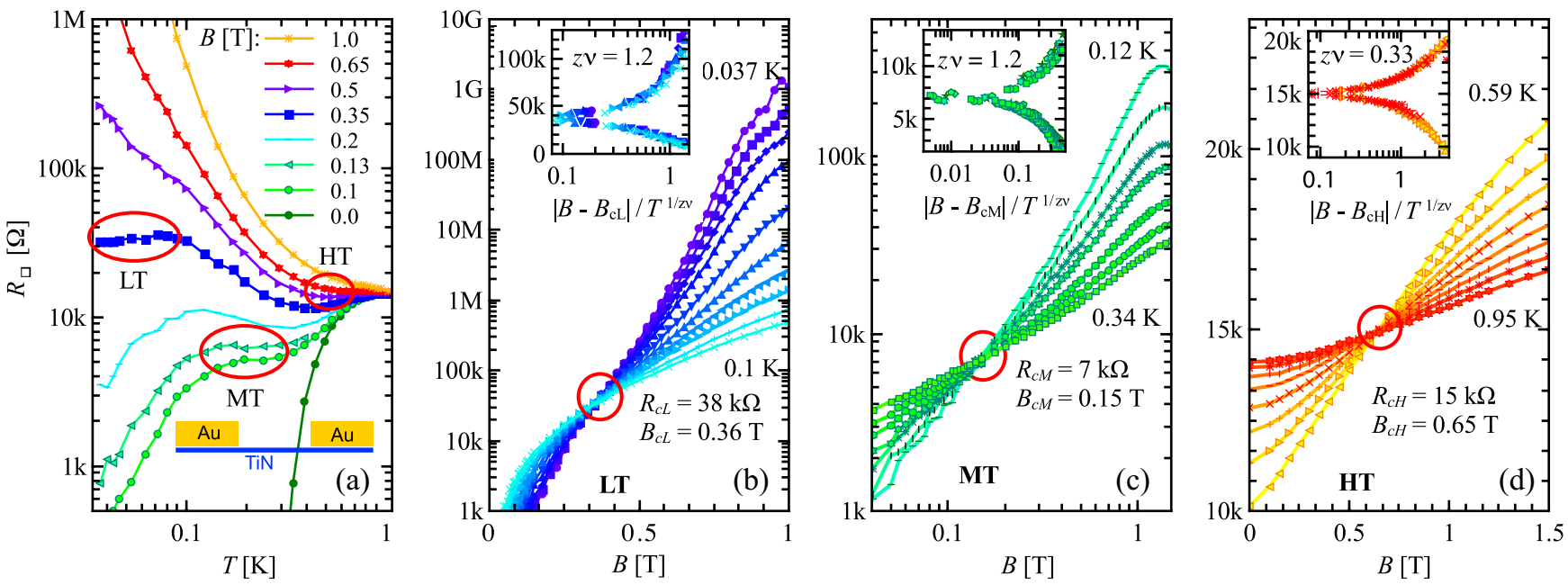

FIG. 2. (a) Temperature dependence of the sheet resistance $R_{\square}(T)$ for several magnetic fields and $R_{\square}^{300}=4.25 \mathrm{k} \Omega$ (chip A). Three plateaus in the $R(T)$ curves at the extrema of $B^{*}(T)$ in Fig. 1 are marked with red ellipses. Inset: schematic of the TiN film with Au contacts. (b) (d) Crossing points in the $R(B)$ isotherms at the plateau regions in (a) corresponding to the extrema in the separatix $B^{*}(T)$ (Fig. 1, left panel). Note that the horizontal scale in (b) and (d) is linear. Insets: scaling analysis for each crossing point. 

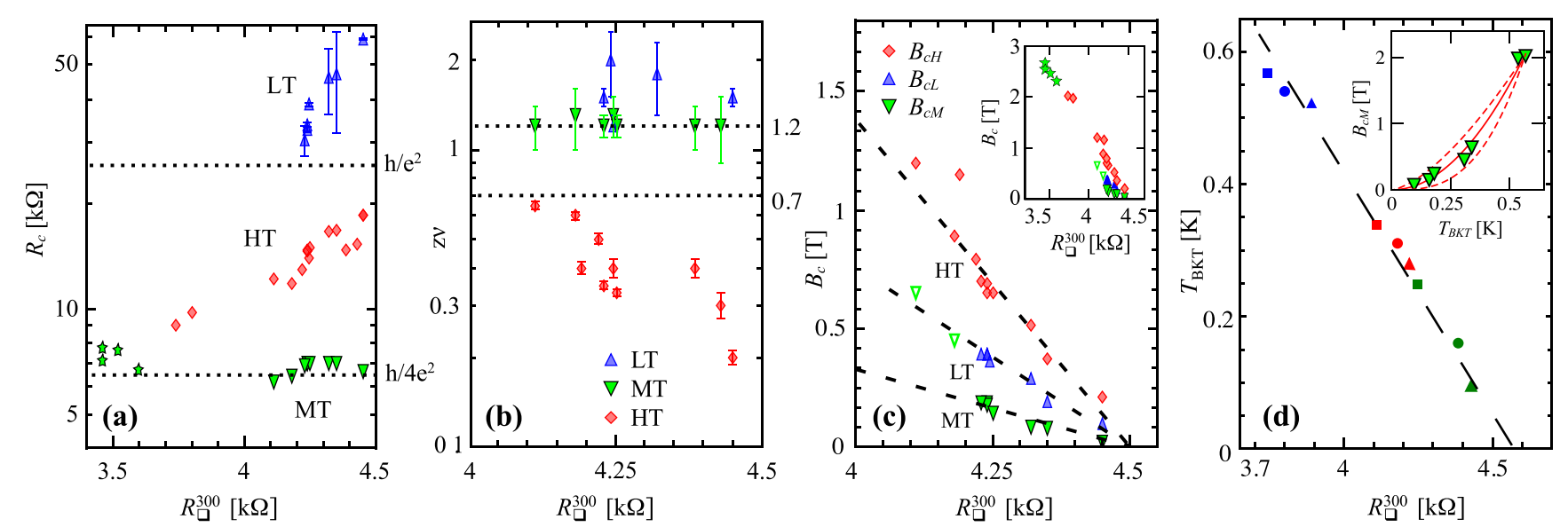

FIG. 3. (a) Critical resistance $R_{c}$ vs sheet resistance at room temperature $R_{\square}^{300}$ (chips A,B). Green stars correspond to disorder levels, for which the MT and HT regimes are merged. (b) Scaling exponent $z v$ vs $R_{\square}^{300}$ resulting from the best data collapse of the $R(B)$ isotherms in the scaling plots [insets of Figs. 2(b)-2(d)]. The values $z v=0.7$ and $z v=1.2$ are marked by horizontal dotted lines. (c) Magnetic fields at the crossing points $B_{c L}, B_{c M}$, and $B_{c H}$ vs $R_{\square}^{300}$. Dashed lines are guides to the eye. Inset: evolution of $B_{c L}, B_{c M}$, and $B_{c H}$ for a wider range of $R_{\square}^{300}$. (d) BKT-transition temperature $T_{\mathrm{BKT}}$ extracted from $R(T, B=0)$ (see text). Inset: $B_{c M}$ vs $T_{\mathrm{BKT}}$. The solid red line corresponds to the exponent $z=1$. The dashed red lines are obtained for $z=4 / 3$ and $z=2 / 3$, respectively.

correlation length $\xi$ characterizes phase fluctuations in the superconductor phase, while the characteristic frequency $\omega$ sets the energy scale for the Berezhinski-Kosterlitz-Thouless (BKT) transition: $k_{\mathrm{B}} T_{\mathrm{BKT}} \propto \xi^{-z}$. This leads to the relation $B_{c} \sim T_{\mathrm{BKT}}^{2 / z}$ [3], which allows for an independent estimate to the dynamical critical exponent $z$. We extracted $T_{\mathrm{BKT}}$ from $R(T, B=0)$ for different films and different oxidation states [20]. The result is shown in Fig. 3(d). It turns out that $T_{\mathrm{BKT}}$ decreases nearly linearly with $R_{\square}^{300}$ and extrapolates to zero at the very same critical value of disorder indicated by $R_{\square}^{300}=$ $4.5 \mathrm{k} \Omega$. In the inset we plot $B_{c M}$ vs $T_{\mathrm{BKT}}$ [inset in Fig. 3(a)] and find $2 / z \simeq 2$, corresponding to $z=1$. This value is associated with long-ranged Coulomb interactions [3].

Unlike the MT regime, we observe disorder-dependent and thus not universal values for $R_{c}$, and $z v$ in the LT and HT regimes. In particular, both $R_{c L}$ and $R_{c H}$ increase with increasing level of disorder (see Fig. 3, left panel). The critical exponent $z v$ in the HT regime is significantly smaller than 1.3 and decreases from $z v \approx 0.7$ to $z v \approx 0.2$ over the investigated range of $R_{\square}^{300}$ (see Fig. 3, middle panel). This is consistent with earlier findings in TiN ( $z v=1,[16])$, but also for $a$-MoGe $(z v=1,[7]), \mathrm{NbSi}(z v=0.67,[23])$, and $a-\mathrm{Bi}(z v=0.7$, [24]) typically in films with $R_{c}<h / 4 e^{2}$. In the LT regime, the values for $z v$ are scattered around a mean $z v=1.6 \pm 0.3$.

\section{INTERMEDIATE $T$-REGIME}

We now compare the properties of our TiN films in the MT regime to earlier observations in amorphous indium oxide films $[8,12,22]$. Similar to our data, values of the critical resistance $R_{c} \simeq h / 4 e^{2}$ and the scaling exponent $z v \simeq 1.2$ are close to those observed in sufficiently resistive $\mathrm{InO}_{x}$, albeit also values of $z v \simeq 2.4$ were reported for $\operatorname{InO}_{x}[25,26]$. In both materials $R(T, B)$ shows an Arrhenius-like behavior in this regime, with a characteristic temperature that changes sign at $B_{c M}$. This sign change indicates the transition from activated transport of vortices to that of Cooper pairs, as suggested in the self-duality picture of Fisher [3]. In addition, the value of $v=1.2$ satisfies the theoretically predicted lower bound of $v \geqslant 1$ for a SIT in a disordered $2 \mathrm{D}$ system [2,3]. The $R(T, B)$ curves are consistent with a power law $\left(B-B_{c M}\right)^{\alpha(T)}$ for $R<$ $R_{c}$ as for $\mathrm{InO}_{x}[12,22,27]$. Deeper in the insulating regime, the behavior crosses over to an exponential dependence on magnetic field [see the upturn in the double logarithmic plot in Fig. 2(c) and the Supplemental Material] that breaks duality symmetry [22].

Our study provides experimental evidence that the scaling exponent $z v \simeq 1.2$ for the magnetic field-induced SIT remains independent of the level of disorder up to the disorderinduced SIT at $B=0$. Since quantum phase transitions fall into certain universality classes, such a disorder-independent $z v$ substantiates the conclusion that the crossing point in the MT regime can be associated with a quantum phase transition. On the other hand, the disorder dependence of $R_{c L}, R_{c H}$, and $z v$ in the LT and HT crossing points reveals a lack of universality and thus discourages their interpretation as quantum critical points.

\section{FLUCTUATION REGIME}

We now analyze the magnetoresistance in the HT regime. For $B \gtrsim 2$ the set of $R(B)$ curves in Fig. 4(a) can be fitted with the Galitski-Larkin theory of superconducting fluctuations in a magnetic field [28], with fixed values of the mean-field transition temperature $T_{c 0}=0.83 \mathrm{~K}$ and upper critical field $B_{c 2}(0)=0.88 \mathrm{~T}$. For magnetic fields above that of the resistance maximum $B_{\max }$, the perturbative theory describes the data rather well, even for low conductances approaching $G_{00}=e^{2} / \pi h \simeq(81 \mathrm{k} \Omega)^{-1}$. In order to adjust the theory to the high field limit, a $B$-independent offset is added that takes into account the normal state conductance $G_{N}$ plus the Altshuler-Aronov (AA) correction $\Delta G_{\mathrm{AA}}(T)$ as the only fitting parameter [20]. The $T$ dependence of the offset in this limit is shown in Fig. 4(b). Its $T$ dependence is logarithmic 

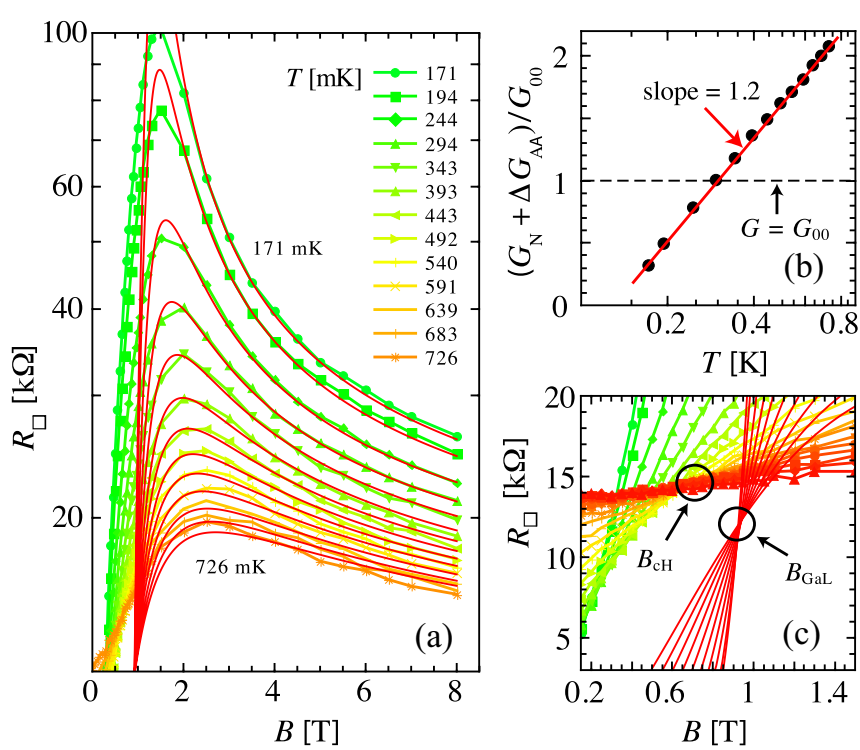

FIG. 4. (a) $R(B)$ isotherms for $R_{\square}^{300}=4.228 \mathrm{k} \Omega$ (chip A). The red lines are fits of Eq. (S5) with the parameters $T_{c 0}=0.83 \mathrm{~K}$ and $B_{c 2}(0)=0.88 \mathrm{~T}$. (b) $G(T)$ extracted from the fits in the limit of large $B$. The red line denotes the Altshuler-Aronov contribution to $G$ (see text). (c) Zoom to the experimental and Galitski-Larkin crossing points $B_{c H}=0.73 \mathrm{~T}$ and $B_{G a L}=0.935 \mathrm{~T}$.

with a prefactor of $1.2 G_{00}$, consistent with the AA correction. At $B<B_{\max }$ the agreement is only qualitative. The theory underestimates the resistance, because it does not contain the flux-flow resistance below $B_{\mathrm{c} 2}$. Nevertheless it reproduces the observed resistance crossing, although at a somewhat higher magnetic field $B=B_{G a L}>B_{\mathrm{c} 2}(T)$ [Fig. 4(c)]. In lower resistance samples $B_{c H}$ and $B_{G a L}$ converge [16,20,21].

The above analysis strongly suggests that the HT plateau in Fig. 2(a) and the corresponding HT crossing point results from an accidental cancellation of different contributions to the $T$-dependent resistance in the interval $0.6 \lesssim T \lesssim 0.95 \mathrm{~K}$, i.e., the interplay between vortex dynamics and the combined Altshuler-Aronov and Galitski-Larkin corrections to $R(T, B)$ [20]. As the crossing of $R(B)$ isotherms is inevitable in the presence of sufficiently strong superconducting fluctuations, we suspect that some of the earlier reports on $R(B)$ crossings may be understood in terms of such an interplay rather than evidencing quantum criticality-in particular, if the sheet resistance at the crossing is low compared to $h / 4 e^{2}$.

\section{LOW T-REGIME}

The crossing point in the LT regime also features disorderdependent $z v$ and $R_{c L}>h / 4 e^{2}$. In order to elucidate its origin, we measured the highly nonlinear $I(V)$ characteristics at low temperatures [Fig. 5(a)]. With increasing voltage we observe current steps in $I(V)$. Similar steps were found in the peculiar insulating state of both TiN and $\mathrm{InO}_{x}$ (termed Cooper pair insulator in Ref. [29]). At higher bias voltage, initially blocked current paths (charge rivers) locally break through within the insulator, leading to the observed steps in $I(V)$ [29-31]. The current steps are the dual counterpart of the voltage steps resulting from the nucleation of vortex rivers in strongly pinned
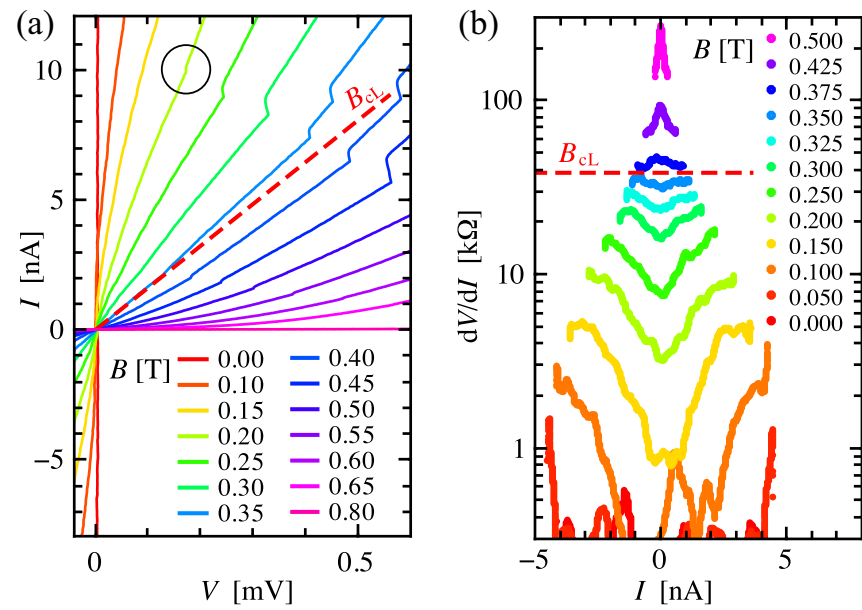

FIG. 5. (a) $I(V)$ characteristics for $T=37 \mathrm{mK}\left(R_{\square}^{300}=4.25 \mathrm{k} \Omega\right.$ chip A). The red dashed line indicates $B<B_{c L}$. The yellow line corresponds to the critical field $B_{c M}$. The black circle indicates first appearance of a (tiny) jump in $I(V)$. The slight left tilt of the jumps results from an imperfect voltage bias, leading to a slight reduction of the voltage after the jump. (b) Corresponding differential resistance $d V / d I$ (right) for $I \lesssim 5 \mathrm{nA}$.

vortex lattices [32]. On the other hand, at $B_{c L}$ and in the low bias regime the differential resistance exhibits a crossover from a superconducting resistance dip to an insulating peak around zero bias [Fig. 5(b)]. The crossing field $B_{c L}$ is indicated by the dashed red lines in Figs. 5(a) and 5(b).

The presence of both resistance dip and current steps in the regime $0.2 \mathrm{~T} \leqslant B \leqslant 0.35 \mathrm{~T}$ signals coexistence of insulating and superconducting regions within the device. The dip in $d V / d I$ is consistent with a percolative network of narrow superconducting filaments embedded into an insulating matrix close to the SIT [33]. The presence of insulating features in $I(V)$ down to $B \lesssim 2$ T further substantiates our above conclusion that in most areas of the film the SIT occurs already at $B_{c M}=0.15 \mathrm{~T}$, and not at $B_{c L}=0.3 \mathrm{~T}$. However, we may indeed have a sequence of two transitions. A first transition takes place at the MT point, where most of our film turns locally insulating in large islands, with small filaments remaining superconducting in between. A second transition could occur at the LT point, where these islands percolate to form a globally insulating state. The film's spatial extension may be just too small to display the universal critical behavior expected in the thermodynamic limit. Further studies are needed to elucidate whether the phenomenology observed in our TiN films applies to other materials too.

\section{CONCLUSIONS}

In conclusion, we have shown that the mere possibility of scaling is insufficient to evidence quantum critical behavior in the vicinity of the SIT. The variation of a second control parameter is mandatory to test for the possible universality of the critical parameters controlling a presumed quantum critical point. Consistent with the above requirement, we demonstrate that the critical exponents $v=1.2$ and $z=1$ in the magnetoresistance of TiN thin films are independent of the 
level of disorder. Disorder-dependent crossing behavior can be induced by inhomogeneity or the compensation of competing contributions to the conductance in certain temperature intervals.

Note added. Recently, we became aware of recent work demonstrating that 2D scaling can also be successfully applied in $1 \mathrm{D}$ wires, supporting our point that scaling alone does not signal the presence of SIT [34].

\section{ACKNOWLEDGMENTS}

We thank M. Baklanov for the supply of TiN material, K. Tikhonov and V. Vinokur for inspiring discussions. T.B. was supported by the A. v. Humboldt Foundation. Work was co-funded by Deutsche Forschungsgemeinschaft (German Science Foundation), Project ID 314695032 SFB 1277 (Subproject B08).
[1] S. L. Sondhi, S. M. Girvin, J. P. Carini, and D. Shahar, Rev. Mod. Phys. 69, 315 (1997).

[2] M. P. A. Fisher, G. Grinstein, and S. M. Girvin, Phys. Rev. Lett. 64, 587 (1990).

[3] M. P. A. Fisher, Phys. Rev. Lett. 65, 923 (1990).

[4] V. F. Gantmakher and V. T. Dolgopolov, Phys. Usp. 53, 1 (2010).

[5] D. B. Haviland, Y. Liu, and A. M. Goldman, Phys. Rev. Lett. 62, 2180 (1989).

[6] A. D. Caviglia, S. Gariglio, N. Reyren, D. Jaccard, T. Schneider, M. Gabay, S. Thiel, G. Hammerl, J. Mannhart, and J. M. Triscone, Nature (London) 456, 624 (2008).

[7] A. Yazdani and A. Kapitulnik, Phys. Rev. Lett. 74, 3037 (1995).

[8] A. F. Hebard and M. A. Paalanen, Phys. Rev. Lett. 65, 927 (1990).

[9] Y. Liu, D. B. Haviland, B. Nease, and A. M. Goldman, Phys. Rev. B 47, 5931 (1993).

[10] N. Marković, C. Christiansen, and A. M. Goldman, Phys. Rev. Lett. 81, 5217 (1998).

[11] V. F. Gantmakher, M. V. Golubkov, V. T. Dolgopolov, A. A. Shashkin, and G. E. Tsydynzhapov, JETP Lett. 71, 160 (2000).

[12] G. Sambandamurthy, A. Johansson, E. Peled, D. Shahar, P. G. Björnsson, and K. A. Moler, Europhys. Lett. 75, 611 (2006).

[13] X. Shi, V. Lin Ping, T. Sasagawa, V. Dobrosavljevic, and D. Popovic, Nat. Phys. 10, 437 (2014).

[14] J. Biscaras, N. Bergeal, S. Hurand, C. Feuillet-Palma, A. Rastogi, R. C. Budhani, M. Grilli, S. Caprara, and J. Lesueur, Nat. Mater. 12, 542 (2013).

[15] M. Diamantini, A. Mironov, S. Postolova, X. Liu, Z. Hao, D. Silevitch, Y. Kopelevich, P. Kim, C. Trugenberger, and V. Vinokur, Phys. Lett. A 384, 126570 (2020).

[16] T. I. Baturina, J. Bentner, C. Strunk, M. R. Baklanov, and A. Satta, Physica B 359-361, 500 (2005).

[17] T. I. Baturina, S. V. Postolova, A. Y. Mironov, A. Glatz, M. R. Baklanov, and V. M. Vinokur, Europhys. Lett. 97, 17012 (2012).
[18] K. S. Tikhonov, G. Schwiete, and A. M. Finkel'stein, Phys. Rev. B 85, 174527 (2012).

[19] S. V. Postolova, A. Y. Mironov, and T. I. Baturina, JETP Lett. 100, 635 (2015).

[20] See Supplemental Material at http://link.aps.org/supplemental/ 10.1103/PhysRevB.103.184512 for (1) Normal state properties of TiN films, (2) Non-linearities of IV-characteristics in the MTregime, (3) Phenomenological expressions for $R(T, B)$, and (4) Superconducting fluctuations in the HT-regime.

[21] V. F. Gantmakher, S. N. Ermolov, G. E. Tsydynzhapov, A. A. Zhukov, and T. I. Baturina, JETP Lett. 77, 424 (2003).

[22] M. Ovadia, D. Kalok, B. Sacepe, and D. Shahar, Nat. Phys. 9 , 415 (2013).

[23] H. Aubin, C. A. Marrache-Kikuchi, A. Pourret, K. Behnia, L. Bergé, L. Dumoulin, and J. Lesueur, Phys. Rev. B 73, 094521 (2006).

[24] N. Marković, C. Christiansen, A. M. Mack, W. H. Huber, and A. M. Goldman, Phys. Rev. B 60, 4320 (1999).

[25] M. A. Steiner, N. P. Breznay, and A. Kapitulnik, Phys. Rev. B 77, 212501 (2008).

[26] N. P. Breznay, M. A. Steiner, S. A. Kivelson, and A. Kapitulnik, Proc. Natl. Acad. Sci. U.S.A. 113, 280 (2016).

[27] A. F. Hebard and M. A. Paalanen, Phys. Rev. Lett. 54, 2155 (1985).

[28] V. M. Galitski and A. I. Larkin, Phys. Rev. B 63, 174506 (2001).

[29] T. I. Baturina, A. Y. Mironov, V. M. Vinokur, M. R. Baklanov, and C. Strunk, Phys. Rev. Lett. 99, 257003 (2007).

[30] T. I. Baturina, A. Bilušić, A. Y. Mironov, V. M. Vinokur, M. R. Baklanov, and C. Strunk, Physica C 468, 316 (2008).

[31] O. Cohen, M. Ovadia, and D. Shahar, Phys. Rev. B 84, 100507(R) (2011).

[32] T. Matsuda, K. Harada, H. Kasai, O. Kamimura, and A. Tonomura, Science 271, 1393 (1996).

[33] T. I. Baturina and V. M. Vinokur, Ann. Phys. 331, 236 (2013).

[34] A. Rogachev and B. Sacépé, Phys. Rev. B 101, 235164 (2020). 